\title{
Development of immersed diffraction grating for the TROPOMI- SWIR spectrometer
}

\author{
A. H. van Amerongen ${ }^{* a}$, H. Visser ${ }^{b}$, R.J.P. Vink ${ }^{b}$, T. Coppens ${ }^{\mathrm{a}}$, R.W.M. Hoogeveen ${ }^{\mathrm{a}}$ \\ ${ }^{a}$ SRON Netherlands Institute for Space Research, Sorbonnelaan 2, 3584 CA Utrecht, The \\ Netherlands \\ ${ }^{\mathrm{b}}$ TNO Science and Industry, Stieltjesweg 1, 2628 CK Delft, The Netherlands
}

\begin{abstract}
We have developed a novel diffraction grating based on lithographical techniques and anisotropic etching in silicon. The grating is designed for the short-wave-infrared channel of the TROPOMI imaging spectrometer that will be launched on ESA's Sentinel 5 Precursor mission to monitor trace gases in the earth atmosphere. Stringent requirements on both the imaging properties and the quality of the spectra translate to a high-tech grating. In our design the dispersion and resolution is increased with a factor 3.4 with respect to conventional gratings by using the grating in immersion, such that diffraction takes place inside the silicon grating material. By lithographic patterning and anisotropic etching of the mono-crystalline silicon we precisely control line spacing and blaze angle. The grating has a line spacing of $2.5 \mu \mathrm{m}$ and is operated in sixth order. We show that an efficiency of $60 \%$ is reached on a $50 \times 60 \mathrm{~mm}^{2}$ grating surface. We compare our test results with numerical calculations for grating efficiency for both polarizations and find good agreement.
\end{abstract}

Keywords: remote sensing, spectral imager, grating, lithography, silicon, immersion

\section{INTRODUCTION}

We have designed and fabricated an immersed grating for the short-wave-infrared (SWIR) channel of the Tropospheric Monitoring Instrument (TROPOMI). This space spectrometer will be launched on ESA's Sentinel 5 Precursor mission to monitor greenhouse gases and pollutants in the earth atmosphere. We define the grating pattern in a silicon substrate using optical lithography. This novel approach has a fourfold benefit over conventional mechanical ruling of gratings: First the gratings are lithographically produced and thus benefit from state-of-the art methods, materials and equipment from the semiconductor industry. Secondly, using anisotropic etching along preferred axes in the silicon crystal arbitrary blaze angles can be obtained enabling optimization of the diffraction efficiency. Thirdly, the etched reflecting facets are very smooth suppressing stray light. A fourth and decisive improvement over traditional gratings is that silicon gratings can be illuminated from inside the medium, or "in immersion", for wavelengths above $1.2 \mu \mathrm{m}$ for which silicon is transparent. The resolution of a grating scales with its size, relative to the wavelength. By illuminating the grating from the inside, as illustrated in Figure 1, the wavelength is reduced by the index of refraction of the medium $n$. Therefore, immersed gratings of high index materials can be made smaller than conventional gratings. The volume gain of the complete spectrometer can be up to $n$-cubed, implying a huge cost reduction for many applications, in particular for space applications. These advantages make the immersed gratings "enabling technology" for future scientific space missions.

The technique of making immersed gratings with anisotropic etching was pioneered by several US, Japanese and European groups. ${ }^{1-6}$ To our knowledge the group of Dan Jaffe at the University of Texas is currently most active with gratings and their applications in ground based astronomy. An alternative method for producing blazed immersed gratings is by mechanical ruling of germanium as studied by Kuzmenko and coworkers. ${ }^{7}$ This approach does not benefit from the advantages of lithography that yield ultimate periodicity control and smooth grooves.

*a.h.van.amerongen@sron.nl; www.sron.nl

Sensors, Systems, and Next-Generation Satellites XIV, edited by Roland Meynart, Steven P. Neeck, Haruhisa Shimoda, Proc. of SPIE Vol. 7826, 78261D · C 2010 SPIE · CCC code: 0277-786X/10/\$18 · doi: 10.1117/12.869018 
The paper is organized as follows: First, the TROPOMI space spectrometer is presented in Section 2. In Section 3 we describe design and the fabrication of the silicon gratings. Test results are presented and compared to simulations in Section 4. We conclude and present an outlook in Section 5

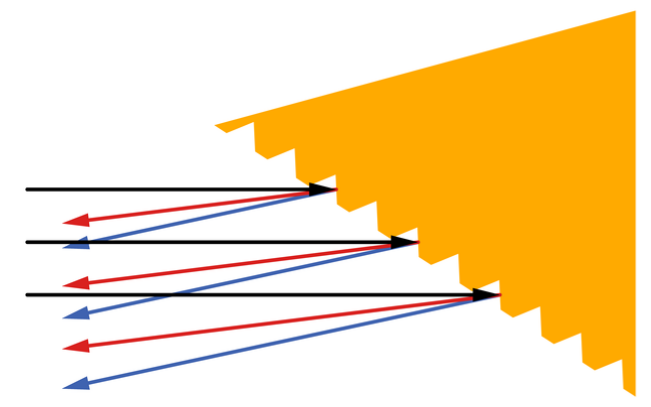

normal grating

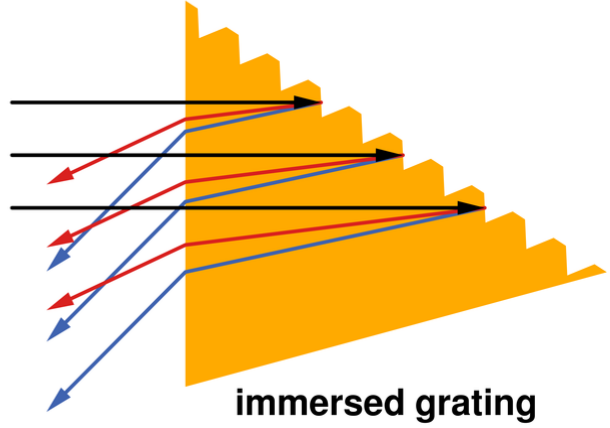

Figure 1. Sketch of the principle of a normal reflection grating (left) and an immersed grating (right), lithographically produced in silicon. The resolving power of the immersed grating is increased with a factor 3.4 of the refractive index as compared to the normal grating. In reality the grating surface is $60 \mathrm{~mm}$ in length and the line spacing is $2.5 \mu \mathrm{m}$.

\section{SPACE SPECTROMETER OPTICAL DESIGN}

The grating development described in this article was initiated on behalf of the space-borne earth-observing atmospheric spectrometer TROPOMI. ${ }^{8}$ TROPOMI has been selected as the only instrument on board ESA's Sentinel-5 precursor mission, to be launched in 2014/2015 as precursor for the ESA Sentinel-5 operational mission, now planned for implementation around 2019. TROPOMI is built on the heritage in design, construction, calibration and data analysis of space-based spectrometers, such as the GOME instrument on ESA's ERS-2 satellite ${ }^{9}$ (launched in 1995), SCIAMACHY $^{10}$ on ESA's ENVISAT (launched in 2002), and OMI ${ }^{11}$ on NASA's EOS-Aura satellite (launched in 2004).

The TROPOMI instrument consists of a nadir-viewing, grating-based imaging spectrometer measuring the spectrum in the ultra-violet to visible spectral range $(270 \mathrm{~nm}-490 \mathrm{~nm})$, in the near infra-red $(710 \mathrm{~nm}-775 \mathrm{~nm})$, and in the SWIR $(2305 \mathrm{~nm}-2385 \mathrm{~nm})$. The instrument will measure both the Earth radiance and solar irradiance spectra, in combination yielding the reflectance spectrum that contains the information for retrieval of atmospheric trace gases. A major improvement with respect to the SCIAMACHY ${ }^{8}$ and OMI ${ }^{9}$ capabilities is the reduced ground-pixel size. This improves the ability to locate sources and sinks of trace gases and increases the number of cloud-free observations. The instrument will measure the main tropospheric pollutants $\left(\mathrm{O}_{3}, \mathrm{NO}_{2}, \mathrm{CO}, \mathrm{CH}_{2} \mathrm{O}\right.$ and $\left.\mathrm{SO}_{2}\right)$ and two major climate gases (tropospheric $\mathrm{O}_{3}$ and $\mathrm{CH}_{4}$ ). In addition, it will measure important parameters of aerosols which play a key role in tropospheric pollution as well as in climate change. The immersed grating development described here will be employed only in the SWIR spectral band.

The TROPOMI instrument design is based on the existing OMI design,${ }^{11}$ where the complete swath of $2500 \mathrm{~km}$ is imaged along the columns of a 2D focal plane array (FPA), while the spectral information is projected along the rows of the detector. Daily global coverage is realized using a sun-synchronous low-earth orbit. The telescope is of a wide-field reflective, telecentric, configuration. The field of view is $108^{\circ}$ across flight and $0.5^{\circ}$ in the flight direction. The satellite scans over the earth as it flies by at $7 \mathrm{~km} / \mathrm{s}$.

The main specifications of the TROPOMI-SWIR spectrometer are given in Table 1. The SWIR optical bench will be cooled to $200 \mathrm{~K}$ to reduce the infra-red background signal and the $\mathrm{HgCdTe}$ focal-plane array will be cooled to about 135 $\mathrm{K}$ to reduce its dark current and noise. These instrument specifications were deduced from the TROPOMI science requirements on the accuracy of the $\mathrm{CO}$ and $\mathrm{CH}_{4}$ data product. The deduction is based on the Iterative Maximum Likelihood Method ${ }^{12}$ for the retrieval the trace gas concentrations. 
Table 1. Main instrument specifications for the TROPOMI-SWIR channel.

\begin{tabular}{|ll|}
\hline Parameter & TROPOMI-SWIR \\
\hline Viewing Geometry & \\
Platform height & $820 \mathrm{~km}$ \\
Swath & $2500 \mathrm{~km}$ \\
Across track spatial sampling & $7 \mathrm{~km}$ \\
Along track spatial resolution & $7 \mathrm{~km}$ \\
Spectrometer parameters & \\
Spectral range & $2305-2385 \mathrm{~nm}$ \\
Spectral resolution & $0.25 \mathrm{~nm}$ \\
Spectral sampling & $0.10 \mathrm{~nm}$ \\
\hline
\end{tabular}

\section{GRATING DESIGN AND FABRICATION}

In this Section we discuss the design of the immersed grating followed by a brief description of the manufacturing process.

\subsection{Grating design}

The size of a spectrometer that meets the requirements of Table 1 is largely determined by the size of the grating. Therefore, the application of an immersed grating strongly reduces the size of the spectrometer. The principle of the gain in size can be explained as follows. Starting from the grating formula:

$$
m \lambda=n d\left(\sin \theta_{i n}+\sin \theta_{m}\right),
$$

where $m$ is the diffraction order, $\lambda$ is the wavelength, $n$ is the refractive index of the medium where the light propagates, $d$ is the line spacing and $\theta_{i n}$ and $\theta_{m}$ are the angles of incidence and diffraction respectively. For a grating in Littrow configuration $\theta_{i n}=\theta_{m}=\theta$, the resolving power is given by:

$$
R=\frac{\lambda}{\Delta \lambda}=\frac{2 n N d \sin \theta}{\lambda} \text {. }
$$

The resolving power is expressed as the ratio of the wavelength and the minimal resolvable wavelength difference $\Delta \lambda$. Note that the term N.d.sin $\theta$ can be regarded as the physical size of the grating. The resolving power scales with $n$, hence the benefit to immerse the grating in a high index medium. This was first recognized by Fraunhofer in 1822, who placed the grating in oil with an index of refraction of about 2. In the SWIR spectral region, transparent media can be used with high index such as germanium $(n=4.0)$ or silicon $(n=3.4)$.

The increased resolving power of an immersed grating is used to scale down the linear size of the grating and thus the diameter of the optical beam. When an equal F-number is used for the camera optics, also the third dimension of the spectrometer scales with $n$. Combined, a reduction in volume of the spectrometer can be obtained of up to $n^{3}$, this is a factor of 64 for germanium and 40 for silicon. Obviously, along with the reduction on volume, also a reduction in mass and cost can be obtained.

We define the grating pattern through photolithography. Subsequently we etch V-shaped grooves, using anisotropic etching as will be explained in Section 3.2. The resulting grating grooves have an apex angle that is determined by the crystal structure of the silicon lattice. The grooves are separated by a flat part "dam" that makes up typically $20 \%-30 \%$ of the total grating period, see Figure 2.

The blaze angle as indicated in Figure 2 has a value of $\delta=\operatorname{ArcTan} \sqrt{ } 2=54.8^{\circ}$. The angle of incidence is $60^{\circ}$. The angles of diffraction in the order -6 are between $47.7^{\circ}$ (for a wavelength of $2305 \mathrm{~nm}$ ) and $52.8^{\circ}$ (for a wavelength of $2385 \mathrm{~nm}$ ). 
Thus the angles of incidence and diffraction are centered on the blaze angle and the grating operates near the optimal Littrow configuration. Outside the medium, the angle difference between incoming and diffracted beams is minimally $25^{\circ}$, leaving enough room for compact positioning of collimating and imaging optics. In Figure 2 the geometry of the immersed grating is sketched in more detail. TM polarization is in the plane of diffraction and TE is directed in the across dispersion direction.

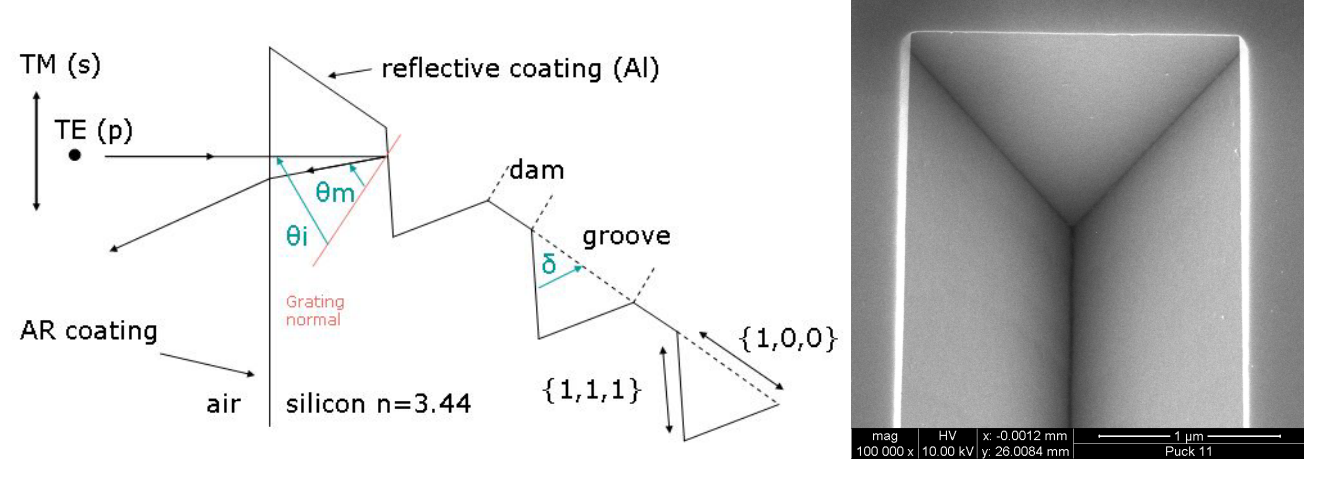

Figure 2. Left: Immersed grating geometry definitions (top view). Right: SEM image of the endpoint of a V-groove.

We have performed numerical simulations of the efficiency of immersed grating models with the PC-grate software, developed by the International Intellectual Group Inc. This software package solves the Maxwell equations for the specific boundary conditions of the grating. The grating design has been optimized using the design parameters line period and dam width. At this point we have chosen to work with silicon substrates that are aligned with the [100] crystal orientation. This leads to symmetric grooves, as shown Figure 2. The freedom to tune the blaze angle was not used for this project. For a different program, gratings have been produced with a blaze angle of $37^{\circ}$, by using silicon substrates that were cut $18^{\circ}$ off the $[100]$ crystal orientation.

The output of the PC-grate program is the optical efficiency as a function of wavelength for the two polarizations with TE (P) polarization being parallel to the grating grooves and TM (S) polarization perpendicular to the grating grooves. The design of the grating is based on the selection criteria: high efficiency, a minimal difference between the efficiency for the two polarization directions, and a small polarization dependence of the efficiency over the wavelength band. The simulations show that the efficiency does not critically depend on the grating period in the range of 4 micrometer down to 1.25 micrometer (diffraction in $10^{\text {th }}$ order to $3^{\text {rd }}$ order respectively). The dam width determines the polarization dependence of the diffraction efficiency although the dependence is not very critical. In the final design we have also taken into account the currently used lithographical production yield that drops for line spacing below $2 \mu \mathrm{m}$ and a dam size below $500 \mathrm{~nm}$. In summary, there is a large parameter range that yields a grating with diffraction efficiency higher than $50 \%$. Aspects of the application and production restrictions determine our choice for $2500 \mathrm{~nm}$ line spacing and a dam width of $800 \mathrm{~nm}$. The physical size of the immersed grating for TROPOMI-SWIR is larger than work previously reported by others ${ }^{6}$ : the grating area is $50 \times 60 \mathrm{~mm}^{2}$, see Figure 3 .

\subsection{Grating fabrication}

The lithographic manufacturing of the immersed gratings involves the following steps. Special $50 \mathrm{~mm}$ high $100 \mathrm{~mm}$ diameter float-zone mono-crystalline silicon substrates with [100] crystal orientation are procured. A $200 \mathrm{~nm}$ thick layer of silicon nitride is applied with low pressure chemical-vapor deposition (LPCVD). In the first lithography step we accurately determine the orientation of the axis of the silicon monocrystal in the plane of the wafer. We reach an accuracy of $\pm 0.05^{\circ}$ by a applying a specialized mask pattern, as developed by Vangbo and Bäcklund ${ }^{10}$. Subsequently, the grating pattern is created: a $700 \mathrm{~nm}$ thick layer of photoresist (AZ ECI 3007) is applied using a home-built spin coater that is adapted for spinning $50 \mathrm{~mm}$ high discs. Illumination of the resist with $300 \mathrm{~nm}$ light is performed under vacuum contact by an adjusted Süss MA-6 mask aligner. A 5 inch photomask contains the grating lines. We have used both laser-written masks and electron-beam written masks. The accuracy of the masks appears to be critical as will be discussed in Section 4.3. After illumination and development of the resist, an fluor-based Reactive-Ion Etcher (RIE) 
opens the silicon nitride layer between the resist lines, exposing the underlying silicon. The grating grooves are etched by a $\mathrm{KOH}$-water solution at $80^{\circ} \mathrm{C}$. The $\mathrm{KOH}$ etching is very anisotropic, with the etch rate typically a factor 60 faster for etching the [100] direction, compared to the [111] direction. As a result, V-shaped grooves are etched with an apex angle of 70.5 degrees as determined by the crystal lattice. The remaining silicon nitride is removed in a 50 mass \% hydrogen fluoride solution. An aluminum reflective coating is sputtered over the grating grooves protected by a $50 \mathrm{~nm}$ thick aliminiumoxide overcoat applied by RF sputtering. Aluminium is preferred above gold as coating material because of its superior adhesion properties on silicon and the higher reflectivity in the applied wavelength band. With the lithography finalized, the prism is cut out of the $50 \mathrm{~mm}$ thick substrate and a multi-layer anti-reflection coating with a reflectivity smaller than $0.5 \%$ is applied on the entrance/exit facet. The prism facets that are not used are grinded with a rms surface roughness of $0.5 \mu \mathrm{m}$. A photograph and a drawing of the immersed grating prism are shown in Figure 3.

\section{PERFORMANCE MEASUREMENTS}

We have tested the gratings in a spectrometer setup that was designed as a breadboard model for the TROPOMI-SWIR spectrometer. ${ }^{8}$ In this Section we describe the three most important aspects of the grating performance: the spectral resolution, the efficiency for both polarizations and the stray light performance.
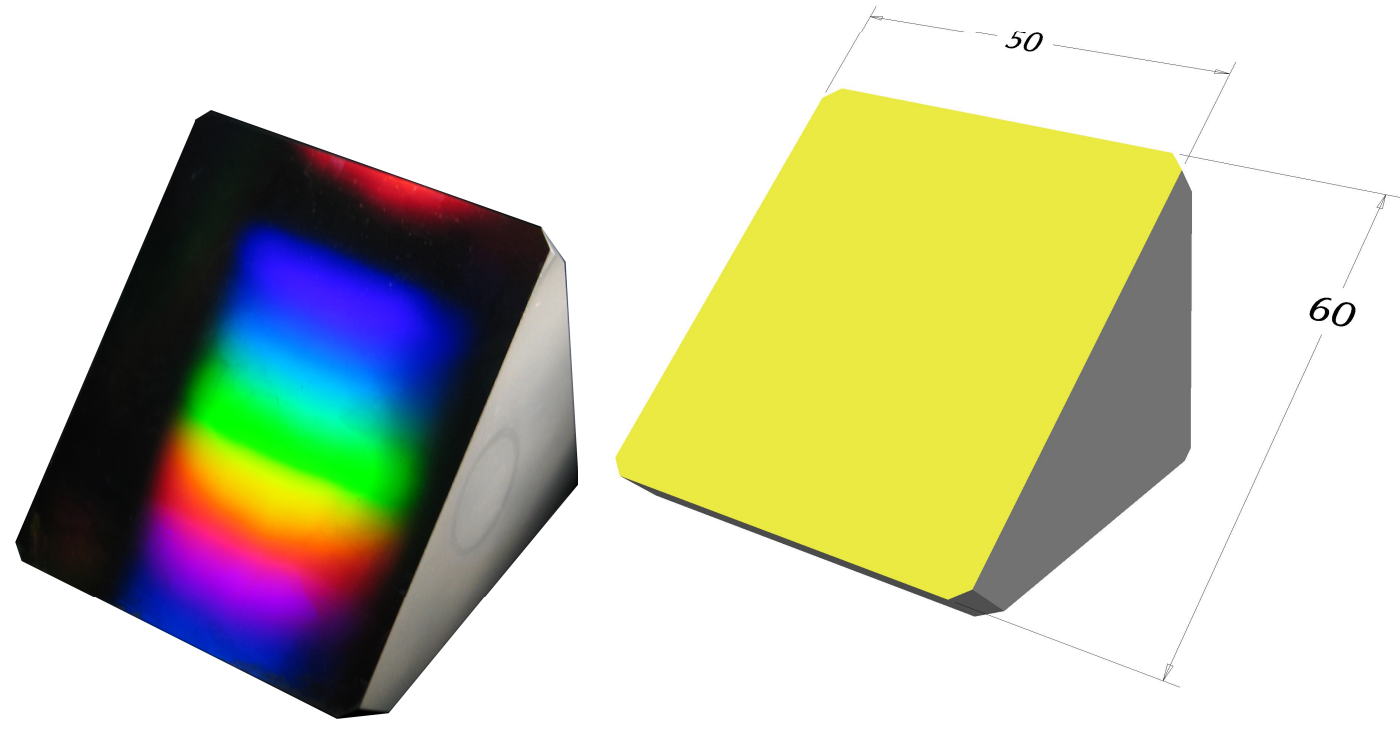

Figure 3. Left: Photograph of monolithical immersed grating. Right: drawing of the grating prism with dimensions in millimeters indicated.

\subsection{Spectral resolution}

The spectral resolution of the TROPOMI spectrometer as well as the resolution of the test setup is designed to be determined by the width of the entrance slit and to a lesser extend by the size of the detector pixels. The spectral resolution is thus not limited by the resolving power of the grating. Therefore we have determined the resolution of the grating indirectly: We have measured the surface-shape error $\left(S S E_{\text {grating }}\right)$ of the grating surface as exposed from the outside (backside) with a Zygo Verifire interferometer operated at $633 \mathrm{~nm}$ in the sixth diffraction order in autocollimation. The resulting angle of incidence for HeNe light in air is close to the angle of incidence for the SWIR light in the silicon medium and therefore the SSE from the outside can be taken as representative for the immersed performance. The $S S E_{f a c e t}$ of the entrance/exit facet is measured in the standard interferometric setup. The total wavefront error (WFE) for the grating as used in immersion can be found from

$$
W F E=\sqrt{\left(2 n S S E_{\text {grating }}\right)^{2}+2\left((n-1) S S E_{\text {facet }}\right)^{2}}
$$


The wave-front error in Equation 3 is composed of the following terms: $S S E_{\text {grating }}$ is multiplied by $2 n$ because the wave is reflected inside the medium, while $S S E_{\text {facet }}$ is multiplied by $n-1$ for a transmission. The beam passes the facet twice, we assume that the errors acquired in the first and second transmission are not correlated. The measured SSE of the gratings that we produce is around $50 \mathrm{~nm}$ rms. This number is governed by imperfections in the uniformity of the lithographic process. The $S S E$ of the entrance/exit facet of the grating is $12 \mathrm{~nm}$ rms in our case. From Equation 3 we find $W F E=345$ $\mathrm{nm}$. This is 0.15 times the operational wavelength of $2345 \mathrm{~nm}$ and approaches the diffraction limit.

\subsection{Efficiency and polarization sensitivity}

The efficiency for both polarization directions of the gratings and its variation across the wavelength band were determined using a modified photospectrometer with a variable detection angle (PerkinElmer Lambda 950). In Figure 4 the measured grating efficiency is compared with simulation results. The dashed lines represent the simulations whereas the continuous lines are the measurement data, limited in resolution by the photodetector noise band of $1 \%$. The measurement agrees very well with the simulations. The measured values for the TE polarization are some $6 \%$ below the simulated value. The averaged efficiency for unpolarized light over the wavelength band is above $60 \%$. Moreover, the polarization sensitivity is low with a variation over the band of only $10 \%$. Discrepancies between simulation and measurement could result from the properties of the reflective coating and the variations in dam width over the grating that were not incorporated in the simulations.

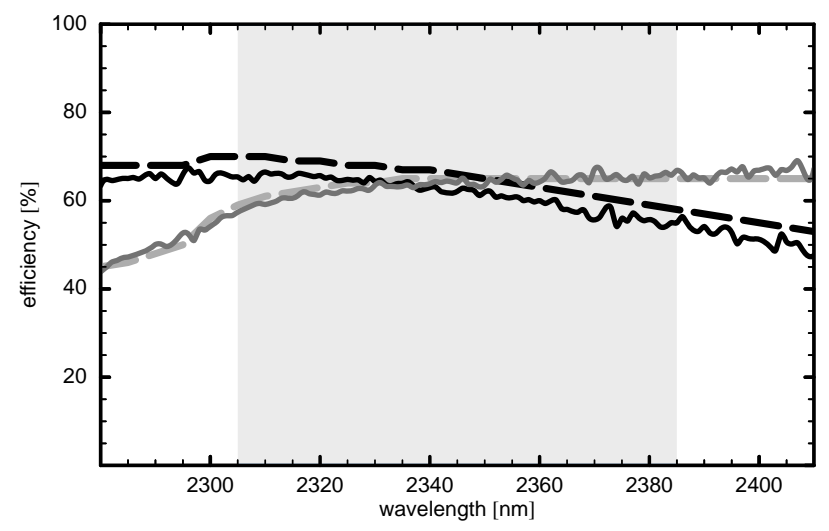

Figure 4. Measured efficiency for the TM polarization (dark gray), TE polarization (black) are compared to simulation results for TM (dashed gray line) and TE (dashed black line). The shaded area indicates the wavelength range of operation.

Typically, the width of the grooves and dams as measured with a scanning-electron-microscope deviate by about $200 \mathrm{~nm}$ from the designed value. The non-uniform dam widths result from the lithography process. We are currently improving our process to improve the uniformity of the dam width over the wafer, which will also improve the WFE.

\subsection{Stray light}

An important figure of merit for a grating is the level of stray light it generates at angles around the diffraction angle. We have measured grating stray-light directly in the spectrometer setup and indirectly by characterizing the surface roughness of our V-groove facets with atomic force microscopy (AFM). The AFM measurements show a rms roughness of 1 to $2 \mathrm{~nm}$, indicating state-of-the-art smoothness. The direct measurement was performed with a narrow band laser (Nanoplus, Germany) as a spectral point source. In the across dispersion direction the entrance slit height is limited in order to reveal the effect of stray light. In Figure 5 a cross-section of the two-dimensional spectral image in both the dispersion and the across-dispersion direction are shown on a logarithmic scale. The 320 pixels in the dispersion direction correspond to a wavelength range of $36 \mathrm{~nm}$. In the across-dispersion direction the complete range of 216 pixels corresponds to the complete swath of 108 degrees. The light intensity falls of rapidly below the $10^{-4}$ of the central intensity. At the $10^{-4}$ level and below modulations in the response can be observed. These modulations, most clearly marked by the peak near pixel 280 in Figure 5 are, so-called, Rowland ghosts, caused by periodic errors in the groove positions. Rowland ghosts result from repetitive errors in the position of the gratings grooves. These placement errors 
originate in the production of the lithographical mask used for the grating definition. We have found that a variety of such periodic errors is present in all masks that are written using a stepping scan-field, both in the masks that were written with a laser beam on the ALTA3000 24 machine at Photronics in the UK and also for two electron-beam written masks produced by Photronics (Germany) and Philips (The Netherlands) respectively. The intensity of a pair of Rowland ghosts of $10^{-4}$ with respect to the parent peak corresponds to a sinusoidal placement error with an amplitude as small as 4 $\mathrm{nm}$. The current stray light performance is very good compared to conventional gratings, nevertheless we aim at suppressing the stray light level even further in the next batch of gratings to be produced by employing a holographically produced grating mask. By virtue of the analog nature of recording such a grating does not have periodic errors.
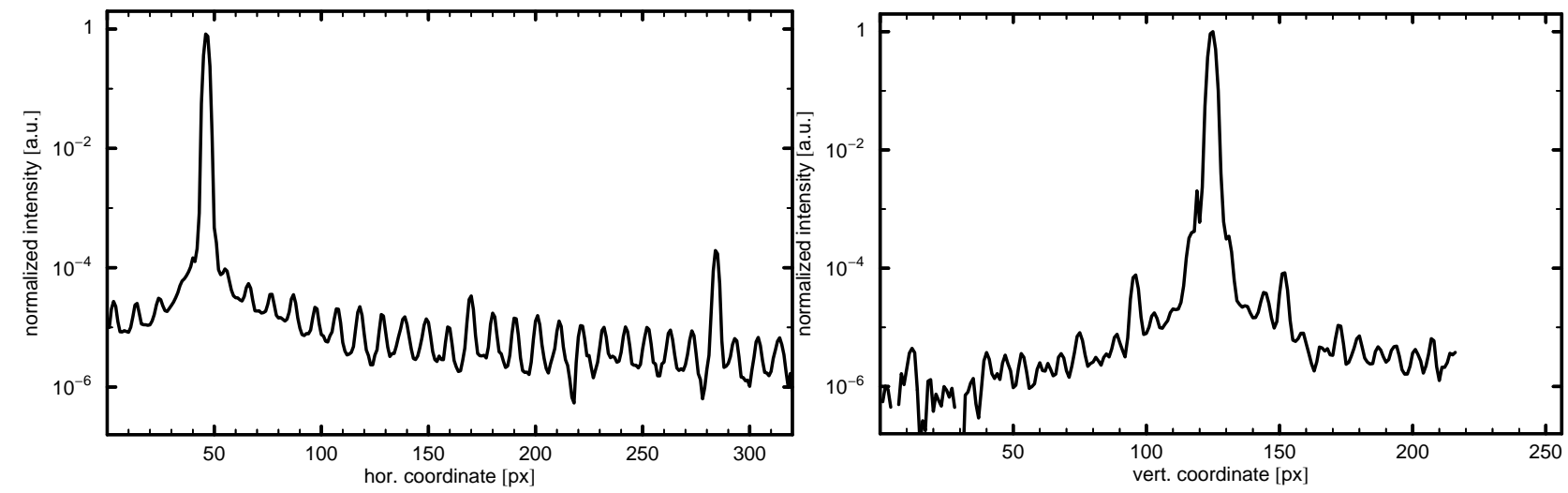

Figure 5. Immersed grating stray light performance in dispersion direction (left) and in across-dispersion direction (right).

\section{CONCLUSION AND OUTLOOK}

We have developed all lithography procedures to produce monolithical immersed gratings to be used in the short-waveinfrared spectral region. The resulting immersed grating is to be employed in the TROPOMI-SWIR spectrometer, selected for space flight on the ESA Sentinel 5 Precursor mission, due to be launched in 2014/2015. The grating has a line spacing of $2500 \mathrm{~nm}$ and a $54.7^{\circ}$ blaze angle. The total grating area is $50 \mathrm{~mm}$ high and $60 \mathrm{~mm}$ in width. Prototype gratings have been tested for efficiency, resolution and stray light. The efficiency is about $60 \%$ and about $5 \%$ under the value derived from simulations. The resolution was shown to be near the diffraction limit with a rms WFE of 0.15 times the operational wavelength. We are currently implementing improvements in the lithography process in order to reach the diffraction limit. Ghosts are on the level of $10^{-4}$ of the parent peak. A next batch of gratings will be produced with a holographically produced grating mask for the reduction of ghosts. With a view on future needs we push our technology towards smaller line widths. Using electron-beam lithography we have successfully etched V-grooves with a line spacing of $400 \mathrm{~nm}$.

\section{ACKNOWLEDGEMENTS}

The work described in this paper was partially funded by the Netherlands Space Office in the PEP framework. We thank Paul Tol for his help with simulations and analysis of the measurement data and Reinder Uuldriks for the mechanical machining and optical coating of the grating prisms. 


\section{REFERENCES}

[1] Graf, U. U., Jaffe, D. T., Kim, E. J., Lacy, J. H., Ling, H., Moore, J. T., and Rebeiz, G., "Fabrication and evaluation of an etched infrared diffraction grating", Appl. Opt., 33, 1, 96-102 (1994).

[2] Kuzmenko, P. J., Ciarlo, D. R., "Improving the optical performance of etched silicon gratings", Proc. SPIE, 3354, 357-367 (1998).

[3] Ebizuka, N., Iye, M., Sasaki, T. and Wakaki, M., "Development of High dispersion grisms and immersion gratings for spectrographs of Subaru Telescope", Proc. SPIE, 3355, 409-416 (1998)

[4] Vitali, F., Cianci, E., Foglietti, V. and Lorenzetti, D., "Toward the fabrication of silicon grisms for high resolution spectroscopy in the near infrared", Mem. S.A.It., 74, 197 (2003).

[5] Ge, J., McDavitt, D., Zhao, B., and Miller, S., "Large format silicon immersion gratings for high resolution infrared spectroscopy", Proc. SPIE, 6273, 62732C (2006).

[6] Marsh, J. P., Mar, D. J., Jaffe, D. T., "Production and Evaluation of Silicon Immersion Gratings for Infrared Astronomy", Appl. Opt., 46, 3400 (2007).

[7] Kuzmenko, P. J., Little, L. M., Davis, P. J., and Little, S. L., "Modeling, fabrication, and testing of a diamondmachined germanium immersion grating", Proc. SPIE 4850, 1179-1190 (2003).

[8] Hoogeveen, R.W.M., Jongma, R.T., Tol, P.J.J., Gloudemans, A., Aben, I.E.E., de Vries, J., Visser, H., Boslooper, E., Dobber, M., Levelt, P.F., "Breadboarding activities of the TROPOMI-SWIR module", Proc. SPIE, 6744, 67441T (2007).

[9] Burrows, J.P. et al., "The Global Ozone Monitoring Experiment (GOME): Mission Concept and First Scientific Results", J. Atmos. Sci. 56, 151-175 (1999).

[10] Bovensmann, H., J.P. Burrows, M. Buchwitz, J. Frerick, S. Noel, V.V. Rozanov, K.V. Chance, A.P.H. Goede, "SCIAMACHY: mission objectives and measurement modes", J. Atmos. Sci. 56, 127-155 (1999).

[11] Levelt, P.F., G.H.J. van den Oord, M.R. Dobber, A. Malkki, H. Visser, J. de Vries, P. Stammes, J.O.V. Lundell, H. Saari, “The Ozone Monitoring Instrument”, IEEE Trans. Geosci. Remote Sens. 44 (5), 1093-1101 (2006).

[12] Gloudemans, A. M. S., H. Schrijver, Q. Kleipool, M. M. P. van den Broek, A. G. Straume1, G. Lichtenberg, R. M. van Hees, I. Aben, and J. F. Meirink, "The impact of SCIAMACHY near-infrared instrument calibration on $\mathrm{CH}_{4}$ and $\mathrm{CO}$ total columns", Atmos. Chem. Phys., 5, 2369-2383 (2005).

[13] Vangbo, M., and Bäcklund, Y., "Precise mask alignment to the crystallographic orientation of silicon wafers using wet anisotropic etching" J. Micromech. Microeng., 6, 279-284 (1996). 